\title{
Improving Word Association Measures in Repetitive Corpora with Context Similarity Weighting
}

\section{Sahala, Aleksi}

SCITEPRESS Science And Technology Publications

2020-11

Sahala , A \& Lindén, K 2020 , Improving Word Association Measures in Repetitive Corpora with Context Similarity Weighting . in A L N Fred \& J Filipe (eds), Proceedings of the 12th International Joint Conference on Knowledge Discovery, Knowledge Engineering and Knowledge Management, IC3K 2020, Volume 1: KDIR, Budapest, Hungary, November 2-4, 2020 . SCITEPRESS Science And Technology Publications, Setúbal , pp. 48-58 , International Joint Conference on Knowledge Discovery, Knowledge Engineering and Knowledge Management (IC3K) , 02/11/2020 . https://doi.org/10.5220/0010106800480058

http://hdl.handle.net/10138/325338

https://doi.org/10.5220/0010106800480058

acceptedVersion

Downloaded from Helda, University of Helsinki institutional repository.

This is an electronic reprint of the original article.

This reprint may differ from the original in pagination and typographic detail.

Please cite the original version. 


\title{
Improving Word Association Measures in Repetitive Corpora with Context Similarity Weighting
}

\author{
Aleksi Sahala and Krister Lindén \\ University of Helsinki, Finland \\ \{aleksi.sahala,krister.linden\}@helsinki.fi
}

Keywords: Collocation Extraction, Distributional Semantics, Computational Assyriology.

\begin{abstract}
Although word association measures are useful for deciphering the semantic nuances of long extinct languages, they are very sensitive to excessively formulaic narrative patterns and full or partial duplication caused by different copies, edits, or fragments of historical texts. This problem is apparent in the corpora of the ancient Mesopotamian languages such as Sumerian and Akkadian. When word associations are measured, vocabulary from repetitive passages tends to dominate the top-ranks and conceal more interesting and descriptive use of the language. We propose an algorithmic way to reduce the impact of repetitiveness by weighting the co-occurrence probabilities by a factor based on their contextual similarity. We demonstrate that the proposed approach does not only effectively reduce the impact of distortion in repetitive corpora, but that it also slightly improves the performance of several PMI-based association measures in word relatedness tasks in non-repetitive corpora. Additionally, we propose normalization for $\mathrm{PMI}^{2}$, a commonly-used association measure, and show that the normalized variant can outperform the base measure in both, repetitive and non-repetitive corpora.
\end{abstract}

\section{INTRODUCTION}

Collocation extraction is a central part of distributional semantics, an area of linguistics that studies meanings of words by observing their cooccurrence patterns. The statistical significance of word co-occurrences can be measured in several different ways. A common idea is to first calculate a chance for some co-occurrence to be independent withing given constraints, and then to compare it with the actual observed co-occurrence probability. The more the observed probability exceeds chance, the stronger the lexical association likely is.

A lot of work has been invested in developing and improving the association measures, especially to correct their bias toward low-frequency events. However, largely unaddressed issue of word association measures concerns their application to corpora with vast amount of repetition or duplication. This problem is apparent in some historical corpora containing very formulaic and repetitive language, as well as slightly diverging versions or editions of same texts. Naturally, for a history researcher it is essential to preserve all existing versions of the documents, but for computational semantic analysis, any full or partial duplicates may give too much weight to certain cooccurrences. In this particular study, we use the ancient Akkadian language as an example, although the issue is also relevant in the Sumerian corpora.

In this paper we propose a metric for measuring contextual similarity within collocation windows, which can be used to weight the co-occurrence probabilities of association measures in order to reduce the impact of repetition without removing any content from the corpus. Our evaluation shows, that the proposed method consistently improves the results in a word relatedness task not only in corpora with repetition, but that it is also slightly advantageous in corpora without noticiable amount of repetition.

We begin this paper with a summary of related work and a short description of the Akkadian language and its resources. Then we give a short review on the different association measures and propose some modifications, which are better compatible with our context similarity weighting (CSW). The last part of the paper will be dedicated to evaluation and discussion. 


\section{RELATED WORK}

Identification of duplicated or reused text has been addressed in multiple publications, especially in the context of file systems (Manber, 1993), web pages (Broder et al., 1997), newspapers (Clough et al., 2002; Smith et al., 2013), plagiarism detection (Gipp, 2014; Citron \& Gingsparg, 2015) and historical corpora (Lee, 2007), but to our knowledge, only one paper has addressed the effect of duplication on distributional semantics. Schofield et al. (2017) measured the effect of duplication on topic-modeling methods, namely LSA (Deerwester et al., 1990) and LDA (Blei et al., 2003). They discovered that LDA is more resistant to duplication if the model is trained with an increased number of topics, and that both models tend to sequester repeated text templates, unless there is not heavy overlapping with topics of interest. Nonetheless, they also suggested that using different deduplication methods such as n-gram removal should have positive impact on the models' performance if the data contains lots of repetition.

Methods of duplicate detection have been widely discussed. Some well-known approaches include approximate fingerprinting (Manber, 1994), Greedy String-Tiling (Wise, 1993) used in plagiarism detection and biology, n-gram overlap (Clough et al., 2002), and w-shingling (Broder et al., 1997), that divides documents or their parts into sequences and measure their resemblance by using Jaccard similarity coefficient. Motivation for our work comes from shingling and n-gram overlap methods.

\section{AKKADIAN AND ITS RESOURCES}

Akkadian was an East-Semitic language documented in hundreds of thousands of cuneiform clay tablets and their fragments excavated from the modern day Iraq and the surrounding regions. Although the earliest attested written sources are dated back to the Old Akkadian Period (2350-2150 BCE), the largest mass of texts and inscriptions were written between $1900 \mathrm{BCE}$ and $500 \mathrm{BCE}$ by the Babylonians and the Assyrians, which both spoke dialects of the Akkadian language. The latest exemplars of Akkadian are dated around $100 \mathrm{CE}$, after which the cuneiform writing tradition disappeared and the language was forgotten until its rediscovery in the middle of the 19th century CE. (Kouwenberg, 2011)
The cultural-historical importance of Akkadian is significant in many respects. First, it was one of the earliest written languages alongside Sumerian, Elamite and Ancient Egyptian. Second, it enjoyed a prestigious status in the ancient Middle-East, and was studied and used in certain contexts by the Hittites, Elamites and the Persians. It was also used as a lingua franca during the Middle-Babylonian period (1590-1100 BCE), for example in Amarna correspondence between Egyptian administration and its representatives in Levant (Streck, 2011). Third, the Akkadian corpus comprises a vast diversity of texts representing many different genres: astronomical texts, administrative and legal documents and law codes, wisdom literature, epics and myths, letters, mathematical texts, lexical and grammatical texts, royal inscriptions, omens, medical texts, as well as several others (Huehnergard \& Woods, 2008). Thus, the Akkadian text material opens an interesting and concrete window to the distant past for a wide range of topics.

Despite being a language that became extinct two millennia ago and studied only by a handful of people, Akkadian is fairly well resourced. Some important digital resources of Akkadian texts are Archives babyloniennes (ARCHIBAB), Cuneiform Digital Library Initiative (CDLI), Sources of Early Akkadian Literature (SEAL), SAAo (State-Archives of Assyria Online) and Open Richly Annotated Cuneiform Corpus (Oracc). Currently only Oracc provides extensive annotation. About $1.5 \mathrm{M}$ tokens of the corpus has been POS-tagged and lemmatized. However, as the corpus covers a time-span of almost 2500 years, it is often not advisable to use it as a whole for linguistic analysis. Instead it is more fruitful to limit the scope to a certain time period or dialect. This limits the size of useable data for studying distributional semantics.

\section{CHALLENGES OF AKKADIAN DATA}

\subsection{Repetition and Repetitiveness}

A challenge concerning the Akkadian text data is its high degree of repetition and repetitiveness. In literary texts, repetition is used as a stylistic feature within single pieces (Groneberg, 1996). Well-known examples can be found in the epic of Gilgameš, for instance, in the description of Gilgameš's travel through the subterranean path of the sun (George, 2003), and the Babylonian creation myth Enüma 
Eliš (Lambert, 2013), where a vizier repeats his master's 53 line long message word by word, which is again almost a word to word repetition of an earlier description of their enemies gathering an army of monsters. Repetitiveness, on the other hand is a genre-defining feature encountered in Assyrian and Babylonian royal inscriptions, divinatory texts and astrological reports (Maul, 2013). Assyrian royal inscriptions, for example, were copied many times over the years with yearly updates and additions of new episodes of that year's campaign. The older passages were often shortened, and the new ones were written closely following the earlier descriptions to promote the stability and prosperity of the Empire (Bach, 2020). Additionally, formulaic epithets contribute to the repetitiveness of the royal inscriptions (Cifola, 1995). Although the copies often show only a slight divergence from each other, Assyriologists consider them as different texts, and they are added into corpora as such.

A part of the repetitiveness, or rather (semi-) duplication, in some Akkadian corpora is also caused by the fragmentary nature of texts. It is not very common that a single fully preserved copy of a text is ever found, especially of larger and linguistically more diverse works. Instead, the Assyriologists often work with small fragments of texts, which they later collate into composite texts. The fragments do not necessarily come from the same place or time period, nor contain the same wording, as copying texts was a central part of the ancient scribal curriculum (Gesche, 2001). Thus, parts of texts may exist in different versions. In Oracc, however, the fragments are not a significant issue, as it mostly consists of edited composites.

When collocation extraction is performed on Akkadian corpora, the formulaic and report-like patterns, extended texts, and to some extent, fragments, tend to distort the association metrics. It is not very rare that even half of the co-occurrences of certain words (including those with rather high co-occurrence frequency) come from identical or almost identical passages.

One option to tackle this issue would be preprocessing the whole corpus as a whole and remove all the "almost duplicate" parts. The disadvantage of this approach would be the need to argue in every case why exactly this part was removed instead of some other. Additionally, drawing a line between too similar and acceptably similar would be arbitrary. Thus, a better and less troublesome approach is not to remove anything, but to reduce the significance of the repeating passages in an algorithmically consistent and reproducible way.
Naturally, it is a valid methodological question to what extent the repetition should be reduced. It is much easier to justify reducing the duplication caused by the fragmentary nature of the corpus, than it is to argue in favor of reducing the impact of the formulaic way of expression. The first mentioned has nothing to do with the language itself, but it is rather a remnant of the scribal training curriculum, evolution of compositions, and unfortunate historical events where tablets or their parts have been damaged or destroyed. The latter, on the other hand, can be considered as a part of the language, at least in its written form.

If we consider this question from the viewpoint of gathering new insights to the Akkadian lexicon, having a look on the freer use of the language by reducing the impact of very obvious associations may be justified. Assyriologists are already well aware of the vocabulary and concepts of the long formulaic litanies, but it is not necessarily obvious that one can see larger patterns in more varied and spread out use of words.

\subsection{Lack of Data}

As a low-resource language, applying machine learning methods such as word2vec (Mikolov et al., 2013) or fastText (Bojanowski et al., 2017) do not necessarily provide outstanding results. Our previous experiments have shown, that in order to get useful results, machine learning approaches have to be applied hundreds of times on the data, and the conclusions must be drawn from the averages (Svärd et al. 2018). Thus it is often more convenient to use count-based approaches such as Pointwise Mutual Information (PMI) (Church \& Hanks, 1990), the results of which can later be transformed into word embeddings by using matrix factorization. In fact, the matrix factorization approach has been shown to be on par (Levy et al. 2015), or even to outperform machine learning methods in low-resource settings, especially in word similarity tasks (Jungmaier, 2020).

Although only a limited number of PMI variants work well with matrix factorization (Levy et al., 2014,2015 ), the variety of useable measures is much greater for collocation analysis. For this reason, we will evaluate our method on several different variants of PMI. 


\section{MEASURING CONTEXT SIMILARITY}

Our method for measuring context similarity involves stacking all the co-occurrence windows of the bigram $(a ; b)$ into a two-dimensional array and calculating the relative frequencies of all unique words vertically in each window position. The context similarity weight (CSW) is calculated as the average of these relative frequencies.

To make each element of the array uniform, we pad the windows to equal length. This keeps the keywords aligned in case they occur near the beginning or end of a line, sentence, paragraph or whatever boundary is chosen to restrict the window span. All padding symbols (\#), the keyword ( $a$ ), and its potential collocate $(b)$, are ignored from relative frequency counts, because they are expected to occur within the same window. Taking them into account would impose a penalty for all cooccurrences.

Formally, over all $n$ co-occurrences of words $a$ and $b$, let $V$ be a set and $W$ a bag or multiset of context words $\{x: x \notin\{a, b, \#\}\}$ that occur at position $i$ in a window of size $w$, and let $m$ be the number of window positions where $V \neq\{\varnothing\}$. We define the context similarity weight $\varphi(a ; b)$ with a magnitude of $k$ as

$$
\varphi(a, b)=\left(\frac{1}{m} \sum_{i=1}^{w} \frac{\left|V_{i}\right|}{\max \left(\left|W_{i}\right|, 1\right)}\right)^{k}
$$

The context words $x$ at position $i$ are perfectly dissimilar if $\left|V_{i}\right|=\left|W_{i}\right|$ and perfectly similar if $\left|V_{i}\right|=$ 1. Thus the bounds for $\varphi$ are $(1 / n)^{k}$ in the case of perfect similarity, and 1 in the case of perfect dissimilarity. The weight can be adjusted by raising it to the power of $k$. Our experiments show that $k$ values of 2 and 3 have generally the best performance in word relatedness task, and that $k$ values higher than 3 are typically detrimental, unless the amount of repetition is very low.

The context similarity weight is applied as a multiplier to the co-occurrence frequency $f(a, b)$, which causes some co-occurrences to be statistically removed from the counts. Thus, the context similarity weighted joint distribution $p(a, b)$ is redefined for a corpus of $N$ words as

$$
p(a, b)=\frac{\varphi(a, b) \cdot f(a, b)}{N}
$$

Applying CSW on the co-occurrence frequencies changes the base definition of PMI and related association measures: a perfect association is no longer determined by two words co-occurring only within a given window, but the context where the words co-occur must also be completely unique. In other words, their distribution is expected to convey previously unseen information.

As the method operates oblivious to the adjacent window positions, it does not systematically take into account deletion or insertion. For this reason it may be useful to remove certain stop words such as articles and prepositions from the corpus altogether to improve the alignment of the windows. The blindness to adjacent positions is merely a safety feature to prevent changes in word order to be considered as exactly same expression. Naturally, attention is also not paid to morphological detail if the text has been lemmatized: for example in English the same sentence in the present and the past tense are considered to contain same information.

One advantage of CSW is that it does not alter the bounds of the association measures, but rather changes their definition. Modifying the observed cooccurrence frequencies can be considered a reordering operation: if the co-occurrence does not include any new information, the words are not removed from the corpus but just considered to exist somewhere else than within the same window. If it provides only some new information, the significance of the co-occurrence is partially reduced. For this reason, the marginal probabilities and the corpus size are not modified.

Calculating the CSW is very fast, as much of it can be done by set operations, which are usually well optimized in programming languages. In terms of space complexity, the method can get heavy if the window size and the corpus are both large. However, this can be reduced significantly by prefiltering stop words and using frequency thresholds.

\section{PMI VARIANTS USED IN EVALUATION}

In this chapter we briefly discuss the properties of common PMI variants we later use in the evaluation. Our aim is to cover variants used in collocation extraction and matrix factorization, as well as measures featuring different frequency biases.

Pointwise mutual information (PMI) was introduced in lexicography by Church and Hanks (1990) by the name association ratio. They defined it as a logarithmic ratio of the observed probability a word co-occurrence within a given window size to 
the expected chance of this co-occurrence under the assumption of independence. The formula itself was based on an earlier definition of mutual information by Fano (1961), but Church and Hanks were the first to apply it on collocation extraction.

$$
\operatorname{PMI}(a ; b)=\log _{2} \frac{p(a, b)}{p(a) p(b)}
$$

After the introduction of PMI, several researchers have proposed various ways to enhance it. The proposed variants generally differ from each other in two respects: in terms of frequency bias and the way scores are bounded and oriented.

Often acknowledged weaknesses of PMI are its sensitivity to low-frequency words (Daille, 1994; Bouma, 2009; Role \& Nadif, 2011; Levy et al., 2015), and its moving upper bound, which makes the scores somewhat unintuitive (Bouma, 2009). In the case of non-co-occurrence, independence and perfect dependence, PMI takes scores of $-\infty<0<$ $-\log _{2} p(a, b)$. The issue of low-frequency bias and the moving upper bound are interrelated. The perfect dependence is achieved when the joint an marginal distributions are equal to each other: $p(a, b)=p(a)=$ $p(b)$, which translates to the denominator being the numerator squared. This means, that a decrease in word frequency increases the value of the upper bound $-\log _{2} p(a, b)$.

Normalized PMI. Bouma (2009) proposed a variant called Normalized PMI (NPMI), which normalized the score orientation to $-1>0>1$ by dividing the score by its moving upper bound.

$$
\operatorname{NPMI}(a ; b)=\log _{2} \frac{p(a, b)}{p(a) p(b)} /-\log _{2} p(a, b)
$$

In addition to providing a more intuitive score orientation, NPMI nullified the effect of an extreme score increase in the case of perfect dependence However, the low-frequency bias reduction of NPMI decreases in practical cases, where the score is not close to the maximum.

PMI $^{\mathbf{k}}$. In terms of low-frequency bias correction, a more robust association measure called $\mathrm{PMI}^{\mathrm{k}}$ was proposed by Daille (1994). The core idea of this measure was to introduce a factor $k$, to which power the $p(a, b)$ is raised to overcome the shrinking denominator problem. The most balanced measure in this family of measures is $\mathrm{PMI}^{2}$ (Evert, 2005), as it preserves the symmetry between the numerator and denominator and keeps the scores consistent regardless of word frequencies.

$$
\operatorname{PMI}^{k}(a ; b)=\log _{2} \frac{p(a, b)^{k}}{p(a) p(b)}
$$

The orientation of $\mathrm{PMI}^{2}$ scores is negative: $-\infty<$ $\log _{2} p(a, b)<0$, which can be generalized for $\mathrm{PMI}^{\mathrm{k}}$ as $-\infty<(k-1) \log _{2} p(a, b)<(k-2) \log _{2} p(a, b)$. This is somewhat problematic, as the score that defines the independence of the co-occurrence is not fixed and the scores are only ranked based on their difference to the perfect association. For this reason, $\mathrm{PMI}^{\mathrm{k}}$ with $k>2$ tends to give high scores for frequently occurring words, regardless if the cooccurrences are statistically dependent or not. This feature makes $\mathrm{PMI}^{3}$ good for finding very general level associations, as demonstrated by Role \& Nadif (2011). $\mathrm{PMI}^{2}$ is less biased toward high frequency words, and it is not very common to see independent co-occurrences in the top ranks if stop words have been removed from the corpus.

Normalized (Positive) PMI ${ }^{\mathbf{k}}$. From the viewpoint of CSW and general readability, it is often more intuitive if the measures feature a fixed threshold for independence as PMI and NPMI do. We propose the following general normalization for $\mathrm{PMI}^{\mathrm{k}}$, which first involves removal of the logarithm and then aligning the threshold of independence with zero. We can fix the upper bound at 1 by following the example of Bouma (2009):

$$
\mathrm{NPMI}^{k}(a ; b)=\frac{\frac{p(a, b)^{k}}{p(a) p(b)}-p(a, b)^{k-1}}{p(a, b)^{k-2}-p(a, b)^{k-1}}
$$

This yields two bounds: 0 for non-co-occurring and independently co-occurring words, and 1 for perfect dependences. The disadvantage of the measure is that co-occurrences rarer than the assumption of independence become unsortable. In fact, cooccurrences may get negative scores as well, but as they approach either the non-co-occurrence or independence, they get closer to 0 . For this reason, it is advisable to use a max-operator as in the popular positive variant of PMI known as PPMI. Thus we define Normalized Positive $\mathrm{PMI}^{\mathrm{k}}$ as

$$
\operatorname{NPPMI}^{k}(\mathrm{a} ; \mathrm{b})=\max \left(\operatorname{NPMI}^{k}(a ; b), 0\right)
$$

Generally the normalization is useful only for $\mathrm{PMI}^{2}$ due to the aforementioned characteristics of $\mathrm{PMI}^{3}$ capturing very high frequency associations, which are not necessarily statistically dependent. For $\mathrm{PMI}^{2}$, the normalization yields slightly better performance in both, context similarity weighted and nonweighted relatedness tasks, as will later be shown in the chapter 8.4.

Some of the PMI variants introduce various constants or discount factors based on word frequencies to balance the frequency distribution. 


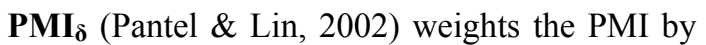
multiplying it with a discount factor $\delta$ defined as

$$
\delta(a ; b)=\frac{f(a, b)}{(f(a, b))+1} \cdot \frac{\min (f(a), f(b))}{\min (f(a), f(b))+1}
$$

The bounds of this variant can be shown to be the same as for the PMI, except for the upper bound at $(f(a, b) /(f(a, b)+1))^{2} \cdot-\log _{2} p(a, b)$. The lowfrequency bias reduction provided by $\mathrm{PMI}_{\delta}$ falls between $\mathrm{PMI}^{2}$ and $\mathrm{PMI}^{3}$.

Semi-Conditional Information weighted with significance of association ( $\mathrm{SCI}_{\text {sig }}$ ) (Washtell \& Markert, 2009) involves first weighing the occurrence probability of the collocate $b$ and multiplying the score with an external factor to reduce the impact of low-frequency bias.

$$
\operatorname{SCI}_{s i g}(a ; b)=\sqrt{\min (p(a), p(b))} \cdot \log _{2} \frac{p(a, b)}{p(a) \sqrt{p(b)}}
$$

The bounds are not mentioned in the original research paper, but they can be shown to exist at $0<$ $\sqrt{p(a, b)}<1$ if $p(a) \leq p(b)$ and $0<p(b)<1$ if $p(a) \geq p(b)$ for non-co-occurring, independent and perfectly dependent events. Alongside $\mathrm{PMI}^{3}, \mathrm{SCI}_{\text {sig }}$ has the highest frequency bias of the measures discussed in this chapter and is thus suitable for finding very general level associations.

Context distribution smoothed PMI (PMI $\alpha$ ) (Levy et al., 2015) is a variant of PMI inspired by negative sampling used in word2vec. This is achieved by raising the $p(b)$ to power of $\alpha$, which is normally set to 0.75 following Mikolov et al. (2013). This measure is among the state-of-art PMI variants for matrix factorization.

$$
\operatorname{PMI}_{\alpha}(a ; b)=\log _{2} \frac{p(a, b)}{p(a) p(b)^{\alpha}}
$$

As an empirical demonstration of frequency distributions of different measures, we scored 1000 random words from ten randomly extracted $10 \mathrm{M}$ token samples of the English Wikipedia corpus and plotted the average frequency of each top- 100 collocate (Figure 1).

PMI, NPMI and $\mathrm{PMI}_{\alpha}$ tend to have the highest bias for low-frequency words, whereas $\mathrm{NPPMI}^{2}$ and $\mathrm{PMI}^{2}$ are more balanced. $\mathrm{PMI}_{\delta}$ falls in the middle between balanced and high-frequency sensitive measures: $\mathrm{PMI}^{3}$ and $\mathrm{SCI}_{\text {sig. }}$.

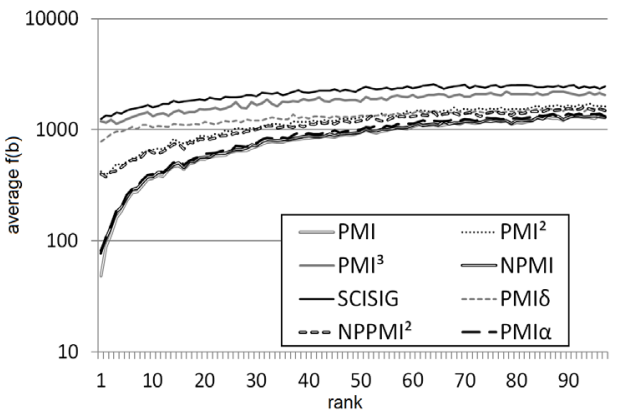

Figure 1: Rank-wise average frequency distributions.

\section{OBSERVATIONS OF CSW ON THE AKKADIAN CORPUS}

Although the effect of CSW cannot be properly evaluated with the Akkadian data due to the lack of a gold standard, we can observe its effect on a very general and subjective level. For these examples we use a symmetric window of seven words and a selection of first millennium BCE texts of various genres comprising 900k tokens.

At first, we examine the top ten ranks of the word nakru 'enemy' by using context similarity weighted PMI $\delta$ with $k$-values of 0 (no CSW), 1 and 3 . The purpose of this test is to demonstrate how the very top ranks are affected when CSW is used. For the sake of simplicity we use the English translations of the Akkadian words.

Table 1: Top-10 collocates of 'enemy' in Akkadian using CSW with different $k$-values.

\begin{tabular}{|c|c|c|c|}
\hline & $k=0$ & $k=1$ & $k=3$ \\
\hline 1 & dangerous & attack & attack \\
\hline 2 & attack & enemy & to attack \\
\hline 3 & enemy & army & enemy \\
\hline 4 & army & to attack & army \\
\hline 5 & weapon & downfall & downfall \\
\hline 6 & *gall bladder & *gall bladder & *gall bladder \\
\hline 7 & *bright & to kill & to kill \\
\hline 8 & to overthrow & to overthrow & border (of land) \\
\hline 9 & *frost & weapon & stranger, outsider \\
\hline 10 & people & *bright & to bind \\
\hline
\end{tabular}

The first observation is, that the number of collocates that seem intuitively strange (marked with asterisks) tend to decrease in this particular case. A closer examination of the corpus reveals that these words mainly come from very repetitive or formulaic contexts. Collocates 'bright' and 'frost' come from astrological reports, which predict an attack by the enemy if certain ominous signs such as 
bright Mars or frost are observed. Word 'gall bladder' comes as well from omen texts. It is, however, preserved because the ominous conditions are more diversely described than the astrological phenomena.

We can also see that two collocates, 'dangerous' and 'people', disappear from the top ranks when the $k$-value is increased, and that the ranking of word 'weapon' decreases. This is due to their appearance in almost identical contexts, as can be seen in the concordance view in Figure 2. We can observe all these words, nakru 'enemy' (here written as a Sumerian logogram ${ }^{\mathrm{LU} 2} \mathrm{KUR}_{2}$ ), bahūlātu 'people', akșu 'dangerous' and GIŠTUKUL 'weapon' in this very context.

Royal Inscriptions of the Neo-Assyrian Period

\begin{tabular}{|c|c|}
\hline & \\
\hline ba-hu-la-ti $\{U R U\} h i-r i m-m e ~\left\{L_{2}\right\} K_{U} R_{2}$ ak-șu & ša ul-tu ul-la a-na \\
\hline ba-hu-la-ti $\{U R U\} h i-r i m-m e ~\left\{L_{2}\right\} K U R_{2}$ ak-șu & ša ul-tu ul-la a-na \\
\hline ba-hu-la-ti $\left\{\right.$ URU\}hi-rim-me $\left\{L_{2}\right\} \mathrm{KUR}_{2}$ ak-și & i-na\{GIŠ\}TUKUL । \\
\hline ba-hu-la-te $\{U R U\}$ hi-rim-me $\left\{L_{2}\right\} K U R_{2}$ ak-și & i-na \{GIŠ\}TUKUL. \\
\hline ba-hu-la-te $\left\{\right.$ URU\}hi-rim-me $\left\{L_{2}\right\} K_{2} U_{2}$ ak-și & i-na \{GIŠ\}TUKUL. \\
\hline ba-hu-la-ti $\left\{\right.$ URU\}hi-rim-me $\left\{L_{2}\right\} K U R_{2}$ ak-și & i-na\{GIŠ\}TUKUL. \\
\hline ba-hu-la-a-ti $\left\{\right.$ URU\}hi-rim-me $\left\{L_{2}\right\} K U F$ & i-na $\{G$ \\
\hline ba-hu-la-ti $\left\{\right.$ URU\}hi-rim-me $\left\{L_{2}\right\} K_{K U R}$ ak-și & i-na \{GIŠ\}TUKUL. \\
\hline ba-hu-la-ti $\{U R U\} h i-r i m-m e ~\left\{L_{2}\right\} K U R_{2}$ ak-șu & \\
\hline
\end{tabular}

Figure 2: Concordance view of repetition in Neo-Assyrian royal inscriptions.

Another interesting detail is revealed if we examine the words associated with a very common word šarru 'king'. If the CSW is not used, the top-10 results are filled with words that have positive connotations: šulmu 'well-being', dannu 'strong one', karābu 'to pray' etc. However, when the $k$ value is increased to 3 , the positive words disappear from the list and collocations with more negative connotations appear to the top ranks. The first ranked collocate is now hamma' $u$ 'rebel' and also a word bǐš 'malicious' appears to the eighth rank of the list. Here, reducing the impact of repetitiveness seems to switch the viewpoint from the Assyrians to their enemies. The reason for this is not very surprising: the Assyrian kings are practically always accompanied with a long litany of praise, and mentioned in very repetitive patterns in royal inscriptions, whereas the enemy kings are just mentioned here and there in more varying contexts.

To experiment numerically how CSW balances the similarity distribution on different $k$-values, we sampled 1000 random words from Oracc, scored them by using PMI $\delta$ and plotted the average context similarity for each rank (Figure 3).

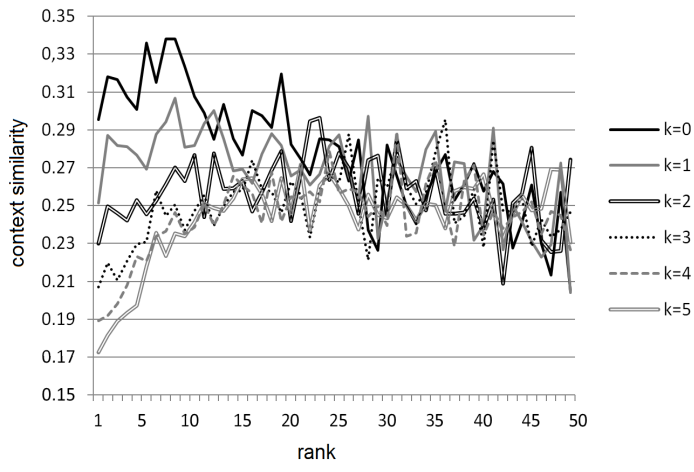

Figure 3: Rank-wise average context similarity.

The figure shows CSW's effect on average context similarity on the top-50 ranks. From the viewpoint of the average context similarity over the whole corpus (0.265), a $k$-value of 2 seems to give the most balanced distribution.

\section{EVALUATION}

\subsection{Test Settings and Parameters}

Because there is no word relatedness gold standard available for the Akkadian language, we experimented on the effect of CSW by generating repetitive versions the English Wikipedia corpus, which we first tokenized and lemmatized using the NLTK (Bird et al., 2009). To get some numerical estimate of general repetitiveness in the Akkadian texts, we sampled 1000 random words from Oracc and measured the average context similarity between them and their collocates in symmetric windows of 3,5 and 7 words. On average, this value was 0.265 , which means that $26.5 \%$ of the context words of any given bigram spanning over an average window of 5.0 are non-unique. For comparison, this figure for the English Wikipedia corpus is only 0.029.

We generated test corpora featuring low, moderate and high degree of repetition, corresponding to average context similarities of $<0.1$, $<0.17$ and $<0.25$ respectively. This process was done by duplicating random segments of the corpus.

To ensure that the test setting was not either favorable or unfavorable by chance, we first extracted ten different random $2 \mathrm{M}$ and $10 \mathrm{M}$ word samples from the Wikipedia corpus. For each evaluation cycle, we generated ten different repetitive versions for each corpus randomly. Thus, for each sample corpus size, the CSW was evaluated on 100 different corpora. 
We scored the WS353 (Agirre et al., 2009) and the Mturk-771 (Halawi et al., 2012) word relatedness test sets using eight different association measures: PMI, NPMI, $\mathrm{PMI}^{2}, \mathrm{PMI}^{3}, \mathrm{NPPMI}^{2}, \mathrm{SCI}_{\text {sig, }}$, PMI $\alpha$ and $\mathrm{PMI} \delta$ with CSW $k$-values between 0 and 3 in symmetric windows of 3,5 and 7 words, and compared the rankings to the gold standard by using Spearman $\rho$-correlation. Our $\rho$-values represent the average correlation over the 100 evaluations for each test setting.

We discarded out-of-vocabulary (OOV) words due to the small sizes of our test corpora. Thus, for the $2 \mathrm{M}$ and $10 \mathrm{M}$ settings, the task was to rank correctly about 30 and 80 words respectively for the WS353, and 35 and 215 words for the Mturk-771 test set. This explains differences between $\rho$ values in different test settings, and also makes the results incomparable between different window and corpus sizes. We did not consider this a problem, because the scope of the evaluation was only to observe, how much CSW contributes to the performance compared to results without it being used.

Due to the large number of association measures included, we choose to discuss in detail only the results for $\mathrm{PMI}_{\delta}$, as is had on average the best performance on the unmodified corpora without CSW. The best performance was measured as follows: we scored all our $2 \mathrm{M}$ and $10 \mathrm{M}$ token base corpora (20 in total) and ranked the measures by their average performance from 1 to $8 . \mathrm{PMI}_{\delta}$ had an average rank of 1.83 , and the other measures $\mathrm{NPPMI}^{2}$ 3.50, $\mathrm{PMI}^{2}$ 4.0, NPMI 4.17, PMI 0 5.0, $\mathrm{SCI}_{\text {sig }}$ 5.33, $\mathrm{PMI}^{3} 5.83$ and PMI 6.33. We will discuss the other measures briefly in chapter 8.3.

\subsection{Overall Performance}

Using CSW generally improves association measures, but the degree of improvement is tied closely to window size and amount of repetition. The overall tendency is that when the window size and amount of repetition increases, the more CSW contributes to the result. This is expected, as larger windows offer a better sample of the surrounding context.

Experiments with unmodified corpora show that CSW provides a slight improvement to the measures on larger windows even if the corpus does not have noticeable repetition. The results for $\mathrm{PMI}_{\delta}$ are summarized in Table 2.

This observation supports our theoretical definition of context similarity weighted association measures mentioned in chapter 5: emphasizing previously unseen contexts over something that has
Table 2: CSW on unmodified test corpora (WS353).

\begin{tabular}{|c|c|c|c|c|}
\hline & No CSW & $k=1$ & $k=2$ & $k=3$ \\
\hline $2 \mathrm{M}-3$ & $\mathbf{0 . 5 5}$ & 0.55 & 0.54 & 0.54 \\
\hline $2 \mathrm{M}-5$ & 0.61 & 0.61 & 0.62 & $\mathbf{0 . 6 2}$ \\
\hline $2 \mathrm{M}-7$ & 0.63 & 0.64 & 0.64 & $\mathbf{0 . 6 5}$ \\
\hline $10 \mathrm{M}-3$ & 0.40 & 0.40 & $\mathbf{0 . 4 0}$ & 0.40 \\
\hline $10 \mathrm{M}-5$ & 0.51 & 0.51 & 0.52 & $\mathbf{0 . 5 2}$ \\
\hline $10 \mathrm{M}-7$ & 0.54 & 0.55 & 0.55 & $\mathbf{0 . 5 6}$ \\
\hline
\end{tabular}

already been observed provides more significant information about the co-occurrence. This hypothesis seems to hold even with small amount of repetition $(r<0.03)$.

Experiments with repetitive corpora show more noticeable improvement in performance. Table 3 shows the improvement in different repetitiveness settings with different parameters. We set the best results from the unmodified corpora (Table 2) as our target scores.

Table 3: CSW on modified test corpora $\mathrm{PMI}_{\delta}$ with different $k$-values (WS353).

\begin{tabular}{|c|c|c|c|c|c|}
\hline & No CSW & $k=1$ & $k=2$ & $k=3$ & Target \\
\hline \multicolumn{7}{|c|}{ Low repetitiveness $(<0.1)$} \\
\hline $2 \mathrm{M}-3$ & 0.48 & 0.51 & $\mathbf{0 . 5 1}$ & 0.51 & 0.55 \\
\hline $2 \mathrm{M}-5$ & 0.54 & 0.58 & 0.59 & $\mathbf{0 . 5 9}$ & 0.62 \\
\hline $2 \mathrm{M}-7$ & 0.55 & 0.59 & 0.61 & $\mathbf{0 . 6 1}$ & 0.65 \\
\hline $10 \mathrm{M}-3$ & 0.39 & 0.40 & 0.40 & $\mathbf{0 . 4 0}$ & 0.40 \\
\hline $10 \mathrm{M}-5$ & 0.48 & 0.50 & 0.52 & $\mathbf{0 . 5 2}$ & 0.52 \\
\hline $10 \mathrm{M}-7$ & 0.52 & 0.54 & 0.55 & $\mathbf{0 . 5 6}$ & 0.56 \\
\hline \multicolumn{7}{|c|}{ Moderate repetitiveness $(<0.17)$} \\
\hline $2 \mathrm{M}-3$ & 0.44 & $\mathbf{0 . 4 8}$ & 0.48 & 0.46 & 0.55 \\
\hline $2 \mathrm{M}-5$ & 0.48 & 0.53 & 0.55 & $\mathbf{0 . 5 5}$ & 0.62 \\
\hline $2 \mathrm{M}-7$ & 0.49 & 0.55 & 0.57 & $\mathbf{0 . 5 8}$ & 0.65 \\
\hline $10 \mathrm{M}-3$ & 0.37 & 0.38 & $\mathbf{0 . 3 9}$ & 0.39 & 0.40 \\
\hline $10 \mathrm{M}-5$ & 0.46 & 0.50 & 0.51 & $\mathbf{0 . 5 2}$ & 0.52 \\
\hline $10 \mathrm{M}-7$ & 0.50 & 0.53 & 0.55 & $\mathbf{0 . 5 6}$ & 0.56 \\
\hline \multicolumn{7}{|c|}{ High repetitiveness $(<0.25)$} \\
\hline $2 \mathrm{M}-3$ & 0.37 & $\mathbf{0 . 3 9}$ & 0.39 & 0.36 & 0.55 \\
\hline $2 \mathrm{M}-5$ & 0.36 & 0.42 & 0.45 & $\mathbf{0 . 4 6}$ & 0.62 \\
\hline $2 \mathrm{M}-7$ & 0.39 & 0.45 & 0.49 & $\mathbf{0 . 5 1}$ & 0.65 \\
\hline $10 \mathrm{M}-3$ & 0.34 & 0.36 & $\mathbf{0 . 3 7}$ & 0.37 & 0.40 \\
\hline $10 \mathrm{M}-5$ & 0.42 & 0.46 & 0.48 & $\mathbf{0 . 4 9}$ & 0.52 \\
\hline $10 \mathrm{M}-7$ & 0.45 & 0.50 & 0.53 & $\mathbf{0 . 5 4}$ & 0.56 \\
\hline
\end{tabular}

Similarly to unmodified corpora, giving too much emphasis on contexts captured by very small windows may have negative impact on the results compared to lower $k$-values. This issue could be solved by using a secondary context window around the actual collocation window.

The CSW is able to reach, or at least to get very close to the target scores in the $10 \mathrm{M}$ setting. This indicates that it effectively reduces or even nullifies the impact of the repetition. In the $2 \mathrm{M}$ setting the target scores are not reached, but the improvement is 
still noticeable in larger window sizes: in the $2 \mathrm{M}-7$ setting the $\rho$ improves 0.09 and 0.12 points in the $r<$ 0.17 and $r<0.25$ settings respectively.

Evaluation using the Mturk-771 test set yields similar results. For reasons of space, the comparison in Table 4 is limited to the average of low, medium and high repetitiveness settings in windows of 5 and 7 scored with $\mathrm{PMI}_{\delta}$.

Table 4: $\rho$-improvement compared to CSW not being used.

\begin{tabular}{|c|c|c|c|}
\hline & $k=1$ & $k=2$ & $k=3$ \\
\hline \multicolumn{3}{|c|}{$10 \mathrm{M}$ corpora $(0.03<r<0.25)$} \\
\hline Mturk-771 & +0.03 & +0.04 & +0.05 \\
\hline WS353 & +0.03 & +0.05 & +0.06 \\
\hline \multicolumn{4}{|c|}{ 2M corpora $(0.03<r<0.25)$} \\
\hline Mturk-771 & +0.03 & +0.05 & +0.07 \\
\hline WS353 & +0.05 & +0.07 & +0.08 \\
\hline
\end{tabular}

\subsection{Measure Specific Improvement}

All measures except $\mathrm{SCI}_{\text {sig }}$ show consistent improvement in performance in all test settings. The reason why CSW is detrimental to $\mathrm{SCI}_{\text {sig }}$ lies likely in the sig-factor, as $\mathrm{PMI}_{\alpha}$ does not show similar performance decrease regardless of its very high similarity to Semi-Conditional Information: these measures are only distinguished from each other by the amount of weight that is given to $p(b)$.

Association measures with low-frequency bias benefit less of the CSW. This is, because lowfrequency bias corrected measures, such as $\mathrm{PMI}^{2}$, modify the weighted joint-distribution amplifying the CSW's effect. Figure 4 summarizes the average improvement of different measures using window sizes of 5 and 7 in the $10 \mathrm{M}$ token corpora. $\mathrm{SCI}_{\text {sig }}$ is excluded from the figure due to its negative performance.

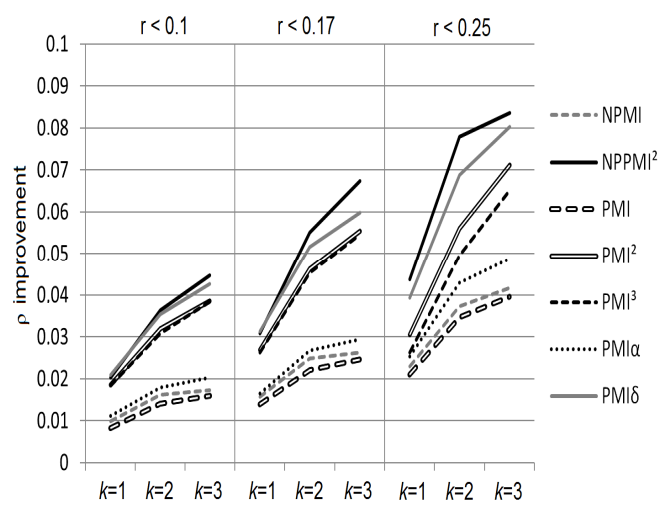

Figure 4: Measure-specific improvement (WS353).
Results gained from the 2M corpora and Mturk-771 test set follow similar distribution.

\subsection{Performance of NPPMI}

Normalizing the $\mathrm{PMI}^{2}$ has positive impact on its performance in all non-repetitive and repetitive test settings. As seen in Figure 4, NPPMI ${ }^{2}$ gains slightly more advantage from CSW than $\mathrm{PMI}^{2}$ does. Table 5 summarizes the difference in performance in the $10 \mathrm{M}$ token corpora using the WS353 test set and a window size of 7 .

Table 5: Performance difference of $\mathrm{PMI}^{2}$ and $\mathrm{NPPMI}^{2}$.

\begin{tabular}{|c|c|c|c|c|c|}
\hline & $k=0$ & $k=1$ & $k=2$ & $k=3$ & Diff \\
\hline \multicolumn{7}{|c|}{$10 \mathrm{M}(r<0.03)$} \\
\hline PMI $^{2}$ & 0.51 & 0.52 & 0.52 & 0.52 & +0.01 \\
\hline NPPMI $^{2}$ & $\mathbf{0 . 5 4}$ & $\mathbf{0 . 5 5}$ & $\mathbf{0 . 5 5}$ & $\mathbf{0 . 5 5}$ & $+\mathbf{0 . 0 1}$ \\
\hline \multicolumn{7}{|c|}{$10 \mathrm{M}(r<0.1)$} \\
\hline PMI $^{2}$ & 0.48 & 0.50 & 0.51 & 0.52 & +0.04 \\
\hline NPPMI $^{2}$ & $\mathbf{0 . 5 1}$ & $\mathbf{0 . 5 3}$ & $\mathbf{0 . 5 5}$ & $\mathbf{0 . 5 6}$ & $+\mathbf{0 . 0 5}$ \\
\hline \multicolumn{7}{|c|}{$10 \mathrm{M}(r<0.17)$} \\
\hline PMI $^{2}$ & 0.46 & 0.49 & 0.51 & 0.51 & +0.05 \\
\hline NPPMI $^{2}$ & $\mathbf{0 . 4 9}$ & $\mathbf{0 . 5 2}$ & $\mathbf{0 . 5 5}$ & $\mathbf{0 . 5 6}$ & $+\mathbf{0 . 0 7}$ \\
\hline \multicolumn{7}{|c|}{$10 \mathrm{M}(r<0.25)$} \\
\hline PMI $^{2}$ & 0.41 & 0.44 & 0.47 & 0.48 & +0.07 \\
\hline NPPMI $^{2}$ & $\mathbf{0 . 4 3}$ & $\mathbf{0 . 4 9}$ & $\mathbf{0 . 5 2}$ & $\mathbf{0 . 5 3}$ & $+\mathbf{0 . 1 0}$ \\
\hline
\end{tabular}

The better performance of $\mathrm{NPPMI}^{2}$ can be explained as a result of subtracting information from statistically less significant co-occurrences, which in conjunction with CSW becomes even less significant in repetitive contexts.

\section{CONCLUSIONS AND FUTURE PLANS}

We introduced a context similarity based weighting for word association measures, which was aimed to improve the results in repetitive corpora. Evaluation of the approach by using artificially repeated random extracts of the Wikipedia corpus indicated that the CSW can reduce the impact of repetitiveness and duplication effectively when larger window sizes are used. We also demonstrated, that CSW can slightly improve the results in a word relatedness task even in corpora which had no noticeable repetition. Thus it seems, that in general reducing the impact of previously seen context information about cooccurrences can improve the performance of association measures.

We also introduced a modification to $\mathrm{PMI}^{2}$, which better takes into account the statistical 
relevance of co-occurrences, and showed a small, yet consistent improvement over the original definition of $\mathrm{PMI}^{2}$.

Although the results gained from CSW may seem very corpus specific, it is likely that there are other similar datasets that may benefit from it. Some examples may be other historical corpora, discussion forum data (consisting of quotes of previous messages) and movie subtitle collections. In general, the advantage of CSW is that it is more resistant to duplicate or semi-duplicate entries in case the corpus is poorly pre-processed.

We only discussed collocation analysis in this paper, but an obvious path for future investigation would be to apply CSW to word embeddings. Our preliminary experiments indicate, that cleaning word vector representations with CSW do improve the results in word similarity tasks, but a more comprehensive evaluation and tests will be required before drawing further conclusions.

\section{ACKNOWLEDGEMENTS}

We acknowledge funding from the Academy of Finland for the Centre of Excellence in Ancient Near Eastern Empires and from the University of Helsinki for the Deep Learning and Semantic Domains in Akkadian Texts Project (PI Saana Svärd for both). We also thank Johannes Bach, Mikko Luukko and Saana Svärd for their valuable feedback, Niek Veldhuis for providing us with the JSON Oracc data and Heidi Jauhiainen for pre-processing it.

\section{REFERENCES}

Agirre, E., Alfonseca, E., Hall, K., Kravalova, J., Pasca, M., Soroa, A., 2009. A Study on Similarity and Relatedness Using Distributional and WordNet-based Approaches. In NAACL-HTL 2009.

Bach, J., 2020 (forthcoming). Untersuchungen zur transtextuellen Poetik assyrischer herrschaftlichnarrativer Texte. SAAS 30.

Bird, S., Loper, E., Klein, E., 2009. Natural Language Processing with Python. O'Reilly Media Inc.

Blei, D. M., Ng, A. Y, Jordan, M. I. 2003. Latent Dirichlet Allocation. In The Journal of Machine Learning Research 3, pp. 994-1022.

Bojanowski, R., Grave, E., Joulin, A., Milokolov, T., 2017. Enriching Word Vectors with Subword Information. In TACL 5, pp. 135-146.

Bouma, G., 2009. Normalized (Pointwise) Mutual Information in Collocation Extraction. In GSCL, pp. 31-40.
Broder, A. Z., Glassman, S. C., Manasse, M. S., Zweig, G. 1997. Syntactic Clustering of the Web. Digital Systems Research Center.

Church, K., Hanks, P., 1990. Word association norms, mutual information and lexicography. Computational Linguistics 16, pp. 22-29.

Cifola, B., 1995. Analysis of Variants on the Assyrian Royal Titulary. Istituto Universitario Orientale.

Citron, D. T., Ginsparg, P., 2015. Patterns of Text Reuse in a Scientific Corpus. In the National Academy of Sciences in the USA.

Clough, P., Gaizauskas, R., Piao, S, S. L., Wilks, Y. 2002. Meter: Measuring Text Reuse. In the $40^{\text {th }}$ Annual Meeting of the ACL, pp. 152-159.

Daille, B., 1994. Approche mixte pour l'extraction automatique de terminologie: statistiques lexicales et filtres linguistiques. $\mathrm{PhD}$ thesis, Université Paris 7.

Deerwester, S., Dumais, S. T., Furnas, G. W., Laudauer, T. K., Harsman, R. 1990. Indexing by Latent Semantic Analysis. In JASIST.

Evert, S., 2005. The Statistics of Word Cooccurrences: Word Pairs and Collocations. $\mathrm{PhD}$ thesis. IMS Stuttgart.

Fano, R., 1961. Transmission of Information: A Statistical Theory of Communications. MIT Press.

Gesche, P. D., 2001. Schulunterricht in Babylonien im ersten Jahrtausend v. Chr. AOAT 275. Ugarit Verlag.

George, A., 2003. The Babylonian Gilgamesh Epic: Critical Edition and Cuneiform Texts. Oxford University Press.

Gipp, B., 2014. Citation-Based Plagiarism Detection: Detecting Disguised and Cross-Language Plagiarism using Citation Pattern Analysis. Springer.

Groneberg, B. 1996. Towards a Definition of Literariness as Applied to Akkadian Literature. In Mesopotamian Poetic Language: Sumerian and Akkadian, Edited by M. Vogelzang, H. Vanstiphout. Groningen, pp. 59-84.

Halawi, G., Dror, G., Gabrilovich, E., Koren, Y. 2012: Large-scale learning of word relatedness with constraints. In KDD 2012, pp. 1406-1414. http://www2.mta.ac.il/ gideon/mturk771.html

Huehnergard, J., Woods, C. 2008. Akkadian and Eblaite. In The Ancient Languages of Mesopotamia, Egypt and Aksum. Edited by R. R. Woodard. Cambridge University Press.

Jungmaier, J., Kassner, N., Roth, B., 2020. DirichletSmoothed Word Embeddings for Low-Resource Settings. In $12^{\text {th }}$ LREC 2020, pp. 3560-3565.

Kouwenberg, B., 2011. Akkadian in General. In Semitic Languages. An International Handbook. Edited by S. Weninger, G. Khan, M. P. Streck, J. C. E. Watson, pp. 330-339. De Gruyter Mouton.

Lambert, G. W., 2013. Babylonian Creation Myths. Eisenbrauns.

Lee, J. 2007. A Computational Model of Text Reuse in Ancient Literary Texts. In he $45^{\text {th }}$ Annual Meeting of the ACL, pp. 472-479.

Levy, O., Goldberg, Y. 2014. Neural Word Embedding as Implicit Matrix Factorization. In Advances in Neural Information Processing Systems, pp. 2177-2185. 
Levy, O., Goldberg, Y., Dagan, I., 2015. Improving Distributional Similarity with Lessons Learned from Word Embeddings. In TACL 3, pp. 211-225.

Manber, U., 1994. Finding Similar Files in a Large File System. In proc. of USENIX Technical Conference.

Maul, S., 2003. Die Wahrsagekunst im Alter Orient: Zeichen des Himmels und der Erde. Beck.

Mikolov, T., Chen, K., Corrado, G., Dean, J., 2013. Efficient Estimation of Word Representations in Vector Space. https://arxiv.org/abs/1301.3781

Pantel, P., Lin, D., 2002. Discovering word senses from text. In the $9^{\text {th }} A C M$ SIGKDD, pp. 613-619. ACM.

Role, F., Nadif, M. 2011. Handling the Impact of Low Frequency Events on Co-occurrence based Measures of Word Similarity: A Case Study of Pointwise Mutual Information. In KDIR 2011, pp. 226-231.

Schofield, A., Thompson, L., Mimno, D., 2007. Quantifying the Effects of Text Duplication on Semantic Models. In EMNLP 2017, pp. 2737-2747.

Smith, D. A, Cordell, R., Dillon, E. M., 2013. Infectious Texts: Modeling Text Reuse in Nineteenth-Centrury Newspapers. In IEEE BigData 2013.

Streck, P. M., 2011. Babylonian and Assyrian. In Semitic Languages. An International Handbook. Edited by S. Weninger, G. Khan, M. P. Streck, J. C. E. Watson, pp. 359-395. De Gruyter Mouton.

Svärd, S., Alstola, T., Sahala, A., Jauhiainen, H. \& Lindén, K. 2018. Semantic Domains in Akkadian Text. In CyberResearch on the Ancient Near East and Neighboring Regions: Case Studies on Archaeological Data, Objects, Texts, and Digital Archiving. Juloux, V. B., Gansell, A. R. \& di Ludovico, A. (eds.). Leiden: Brill.

Washtell J., Markert, K., 2009. A comparison of windowless and window-based computational association measures as predictors of syntagmatic human associations. In EMNLP, pp. 628-637.

Wise, M. J. 1993. Running Karp-Rabin Matching and Greedy String Tiling. Basser Department of Computer Science Technical Report 463.

\section{RESOURCES}

ARCHIBAB, 2008. Archives babyloniennes. http://www.archibab.fr/

CDLI, 2000. The Cuneiform Digital Library Initiative. https://cdli.ucla.edu/

Oracc, 2014. The Open Richly Annotated Cuneiform Corpus. http://oracc.org/

SAA(o), 1986. State Archives of Assyria (Online). http://www.helsinki.fi/science/saa/

SEAL, 2008. Sources for Early Akkadian Literature. https://www.seal.uni-leipzig.de/

The Wikipedia Corpus, (downloaded 2019-01-28). https://www.english-corpora.org/wiki/ 